\title{
Mathematical modeling of thermal technical characteristics of external protections with air layers
}

\author{
Rustam Mansurov, Tatyana Rafalskaya* and Dmitry Efimov \\ Novosibirsk State University of Architecture and Civil Engineering (Sibstrin), Russia, 630008 \\ Novosibirsk, Leningradskaya St., 113
}

\begin{abstract}
A screened enclosing structure of the outer wall of the building without the use of thermal insulation materials is proposed. To ensure the required thermal resistance of the outer fence, screens (cement-containing slabs) and closed air gaps between them are used. Using the ANSYS software package, the heat transfer processes were modeled in a shielded building envelope, consisting of the main (carrier) part and screens with air gaps closed between them. The features of the heat transfer process in the thickness of the outer enclosure are revealed.
\end{abstract}

\section{Introduction}

Problems of the rational use of fuel and energy resources, new technologies for the construction of pre-fabricated structures make the actual task of insulating the facades of buildings, both reconstructed and newly built. Traditional multi-layer enclosing structures consist of supporting (structural) and insulating layers. Various types of thermal insulation materials are used, which, as a rule, should be located on the outside of the fence. Mineral wool, polystyrene foam, polyurethane foam, etc. are currently used as thermal insulation layers. The following facade structures are widely used: sandwich panels, ventilated facades, etc. The choice of thermal insulation material is influenced by their characteristics: density, thermal conductivity, heat absorption of the surface. Thermal insulation materials are a weak link in external enclosing structures, since they have several disadvantages: combustibility, hygroscopicity, shrinkage, poor noise insulation, low strength, etc. It is known that the most effective heat insulator is air in a stationary or inactive state [1], for example, in closed air spaces. Back in 1905, V.M Chaplin proposed to use air gaps as a heat-insulating material in enclosing structures [2]. As a result of the research he showed that:

- $\quad$ an alternative to a single layer of insulation can serve several air gaps;

- $\quad$ optimal air gap width is $25-50 \mathrm{~mm}$;

- air gaps should not be made high to reduce the rate of air circulation in them, if possible, cross them in height by horizontal partitions;

\footnotetext{
* Corresponding author: rafalskaya.ta@yandex.ru
} 
- air spaces should not be connected with each other and with the surrounding space, as this impairs their thermal insulation properties.

Despite the fact that these studies were performed more than 100 years ago, previously shielded walling structures were not used in construction without the use of thermal insulation materials.

The aim of the work is computer modelling in the ANSYS software package of enclosing structures that do not use thermal insulation materials. Previously, a similar approach was successfully used for the thermal engineering calculation of a multi-layer enclosing structure of the "sandwich panel" type [3].

\section{Formulation of the problem}

In work models of the panels containing one and 15 air gaps are considered. The fig. 1 shows a cross section of a walling structure with one air gap (model 1). Numbers 1-6 marked the number of layers, the characteristics of which are shown in table 1.

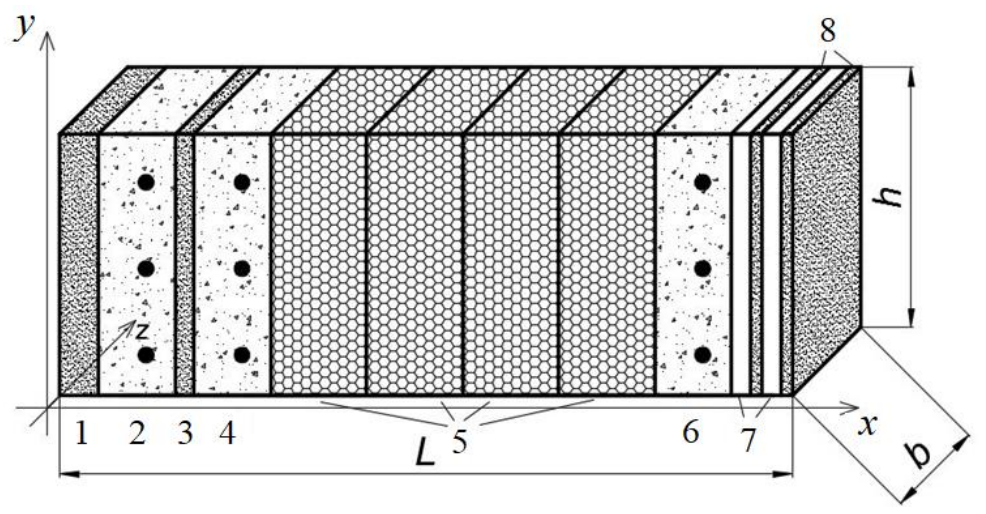

Fig. 1. Scheme of the model number 1. Fencing design with one air gap

Results of natural tests are resulted in Table 1.

Table 1. Characteristics of the enclosing structure by layers

\begin{tabular}{|c|c|c|c|c|c|}
\hline $\begin{array}{c}\text { Layer } \\
\text { number }\end{array}$ & Material & $\begin{array}{c}\text { Heat } \\
\text { conductivity } \\
\text { coefficient } \lambda, \\
\mathbf{W} /(\mathbf{m} \cdot \mathbf{K})\end{array}$ & $\begin{array}{c}\text { Layer } \\
\mathbf{T h i c k n e s s} \\
\boldsymbol{\delta}, \mathbf{m m}\end{array}$ & $\begin{array}{c}\text { Density } \\
\boldsymbol{\rho}, \mathbf{k g} / \mathbf{m 3}\end{array}$ & $\begin{array}{c}\text { Specific } \\
\text { heat } \\
\text { capacity } \boldsymbol{c}_{\boldsymbol{p}}, \\
\mathbf{k J} /(\mathbf{k g} \mathbf{K})\end{array}$ \\
\hline 1 & Plaster & 0.7 & 10 & 1600 & 0.84 \\
\hline $2,4,6$ & Reinforced concrete & 1.92 & 30 & 2500 & 0.84 \\
\hline 3 & Concrete mortar & 0.76 & 5 & 1800 & 0.84 \\
\hline 5 & Styrofoam & 0.052 & 200 & 10 & 1.34 \\
\hline 7 & Air gap & $0,14 \div 0,28$ & 16 & 1.225 & 1.005 \\
\hline 8 & $\begin{array}{c}\text { Shield (Cement } \\
\text { Plate) }\end{array}$ & 0.47 & 8 & 1800 & 0.84 \\
\hline
\end{tabular}

If we assume that the temperature distribution is the same in all cross sections, the problem can be considered as two-dimensional. If, in addition, to neglect the dependence of 
temperature on the vertical coordinate, we obtain a one-dimensional approximation of the problem.

The fig. 2 shows a 3D model of a building envelope with 15 air gaps (model No. 2). Layers 1-8 are the same as in model No. 1 . In the 3D formulation, the problem was solved taking into account the movement of air.

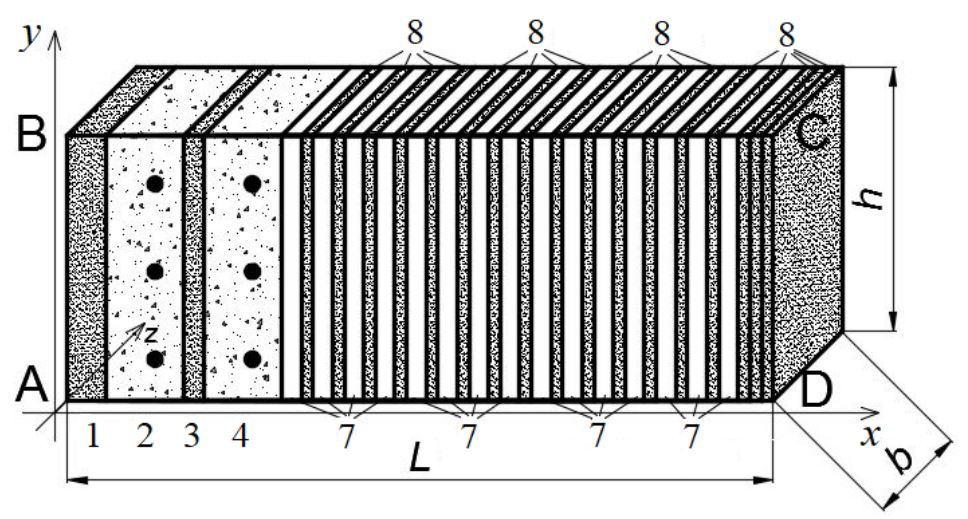

Fig.2. Scheme of model No. 2. 3D model of a building envelope with 15 air gaps

\section{Mathematical models and methods of solution}

The simulation of thermal processes in a multi-layer enclosing structure was performed in one-, two- and three-dimensional productions using our own program in the MathCAD package and various ANSYS PC modules.

\subsection{Approach 1}

To describe the processes of heat transfer in a multi-layer building envelope in a onedimensional formulation, the $1 \mathrm{D}$ heat conduction equation is used:

$$
\rho \cdot c_{p} \cdot \frac{\partial T}{\partial t}=\frac{\partial}{\partial x}\left(\lambda \frac{\partial T}{\partial x}\right)+F(x, t)
$$

where $F(x, t)$ - function describing heat sources or sinks. Equation (1) can be written in the form:

$$
\frac{\partial T}{\partial t}=\frac{\partial}{\partial x}\left(K(x) \frac{\partial T}{\partial x}\right)+F(x, t)
$$

where $K(x)=\lambda(x) /\left[C_{p}(x) \cdot \rho(x)\right\rfloor$ - material thermal diffusivity, $K>0$.

The values of coefficients of thermal diffusivity of materials of different layers of the structure are given in table 2.

Table 2. Thermal diffusivity values.

\begin{tabular}{|c|c|c|}
\hline Layer number & Material & Thermal diffusivity $\mathbf{K}, \mathbf{~ m}^{\mathbf{2}} \mathbf{c}$ \\
\hline 1 & Plaster & $5.6 \cdot 10^{-7}$ \\
\hline $2,4,6$ & Reinforced concrete & $8.1 \cdot 10^{-7}$ \\
\hline
\end{tabular}




\begin{tabular}{|l|c|c|}
\hline 3 & Concrete mortar & $5.6 \cdot 10^{-7}$ \\
\hline 5 & Styrofoam & $1.8 \cdot 10^{-5}$ \\
\hline 7 & Air gap & $6.7 \cdot 10^{-7}$ \\
\hline 8 & Shield (Cement Plate) & $5.6 \cdot 10^{-7}$ \\
\hline
\end{tabular}

Equation (1) is solved by the setting method in the domain $D=\left[0 ; T_{k}\right] \times[0 ; L]$, where $L-$ panel thickness, $T_{k}$ - final moment of time. At the moment $t=0$, the initial temperature distribution $T=0^{\circ} \mathrm{C}$ is set. At $x=0$ (inner surface) and $x=L$ (outer surface), boundary conditions of the 3 rd kind (convective heat exchange) are set:

$$
-\left.\lambda \frac{\partial T}{\partial n}\right|_{w}=\alpha\left(T_{w}-T_{\infty}\right)
$$

where $T_{w}$ - wall temperature, ${ }^{\circ} \mathrm{C} ; T_{\infty}-$ ambient temperature, ${ }^{\circ} \mathrm{C}$.

The calculations used the values of heat transfer coefficients for the inner surface of the building envelope $\alpha_{i n}=8.7 \mathrm{~W} /\left(\mathrm{m}^{2} \mathrm{~K}\right)$, the outer surface $\alpha_{\text {out }}=23 \mathrm{~W} /\left(\mathrm{m}^{2} \cdot \mathrm{K}\right)$. Set temperatures $T_{\infty, i n}=21^{\circ} \mathrm{C}$ for the inner surface $(x=0)$ and $T_{\infty, \text { out }}=-37^{\circ} \mathrm{C}$ for the outer surface $(x=L)$.

We construct in the region $\mathrm{D}$ a finite difference grid with steps $\tau$ and $\mathrm{h}$. To solve the $1 \mathrm{D}$ problem, we use an implicit finite-difference scheme with a special approximation of heat fluxes, which makes it possible to calculate an equation with a discontinuous thermal diffusivity [4].

$$
\frac{T_{j}^{n+1}-T_{j}^{n}}{\tau}=\frac{1}{h^{2}} \cdot\left[\frac{K_{j}^{n}+K_{j+1}^{n+1}}{2}\left(T_{j+1}^{n+1}-T_{j}^{n+1}\right)-\frac{K_{j}^{n}+K_{j-1}^{n}}{2}\left(T_{j}^{n+1}-T_{j-1}^{n+1}\right)\right],
$$

For approximation of the boundary conditions (2) uses the relations:

$$
\begin{gathered}
\lambda_{\text {вн }} \frac{T_{1}^{n+1}-T_{0}^{n+1}}{h}=\alpha_{\text {вн }}\left(T_{0}-T_{\infty, \text { вн }}\right) ; \\
-\lambda_{\text {нар }} \frac{T_{M}^{n+1}-T_{M-1}^{n+1}}{h}=\alpha_{\text {нар }}\left(T_{M}-T_{\infty, \text { нар }}\right) .
\end{gathered}
$$

Rewrite (3) as a three-point equation:

$$
A A_{j} \cdot T_{j-1}^{n+1}-C C_{j} \cdot T_{j}^{n+1}+B B_{j} \cdot T_{j+1}^{n+1}=F F_{j},
$$

were $\quad A A_{j} \cdot=\frac{K_{j}+K_{j-1}}{2} \cdot \frac{\tau}{h^{2}} ; \quad B B_{j} \cdot=\frac{K_{j}+K_{j+1}}{2} \cdot \frac{\tau}{h^{2}} ; \quad C C_{j} \cdot=A A_{j}+B B_{j}+1 ;$ $A F F_{j}=-T_{j}^{n}$.

The sweep method [5] is used to determine the solution on the upper time layer $n+1$.

\subsection{Approach 2}

The heat transfer processes for models 1 and 2 of the two and three-dimensional approximation were calculated in the StaticThermal and Fluent modules ANSYS.

General view of the 2D/3D heat equation: 


$$
\frac{\partial T}{\partial t}=\sum_{i=1}^{N} \frac{\partial}{\partial x_{i}} K(x) \frac{\partial T}{\partial x_{i}}+Q,
$$

where $N=2$ and 3 - for two and three-dimensional problems, respectively.

In a two-dimensional formulation, a panel with a height of $1 \mathrm{~m}$ was considered. On the side surfaces $x=0$ and $x=L$, the conditions for convective heat transfer are set, similar to (2). At the upper and lower boundaries $A D, B C$, the heat insulation conditions are set (no heat flow).

When solving a three-dimensional problem, a panel with a height of $1 \mathrm{~m}$ and a width of $1 \mathrm{~m}$ was considered. The thermal insulation conditions are set on all lateral surfaces of the Symmetry type:

$$
\left.\frac{\partial T}{\partial n}\right|_{w}=0
$$

On the external and internal surfaces - the conditions of convective heat transfer (2).

This approach allows to simulate stationary thermal processes in solids and in air spaces. However, the effects of convective heat transfer due to the movement of air in air spaces, which can make a significant contribution to the thermal resistance of the structure, are not taken into account in this approach. Therefore, in the next step, the task was modeled in the ANSYS Fluent module.

\subsection{Approach 3}

The mathematical model, in addition to the heat equation, includes the Navier - Stokes equations [6], which describe the movement of air. Therefore, the model allows to take into account the convective movement of air in the layers. Since the flow velocities are small, the calculation was performed on the assumption of laminar flow. Accounting for gravity and the dependence of air density on temperature is necessary for the organization of natural convection in confined spaces.

For a full-fledged study, the calculation was also carried out taking into account radiant heat transfer, for which the source term $Q$, which is determined by the Stefan-Boltzmann law, was added to the right side of the equation:

$$
Q=q_{\text {л }}=\varepsilon \cdot \sigma \cdot\left(T_{\infty}^{4}-T_{w}^{4}\right),
$$

where $\varepsilon$ is the emissivity of the surface (degree of blackness); $\sigma$ is the constant of Stephen Boltzmann.

$\varepsilon=0.4$ - which corresponds to the accepted material (aluminum foil). $\varepsilon$ In further calculations, the value of the degree of blackness of the screens is taken to be

The air has a low absorbing and dispersing ability. In this case, the thermal radiation of the medium itself can be neglected, and the radiant heat transfer is calculated between the surfaces bounding this medium, so the S2S model (SurfacetoSurface) was chosen to calculate the radiant heat transfer [7].

To assess the effectiveness of walling, use the value of thermal resistance, which characterizes the property of matter to resist temperature changes.

$$
R_{0}=\frac{1}{\alpha_{\mathrm{B}}}+\sum R_{i}+\frac{1}{\alpha_{\mathrm{H}}},
$$

Here $R_{i}=\delta_{i} / \lambda_{i}$ is the thermal resistance of the $i$-th layer of the structure; $\delta$ - thickness of the building envelope, $\mathrm{m}$. 


\section{Results and discussions}

The solution of the problem for model No. 1 was obtained using approaches 1 and 2 . At the initial moment, the wall had a temperature $T=0^{\circ} \mathrm{C}$. Calculation "on the establishment of" made by the methods described above. The number of grid elements in approach 2 was 11,500 elements. In approach 1 , the difference grid step $h=0.2 \cdot 10^{-3}$ was used. The calculation results are presented in Fig. 3.

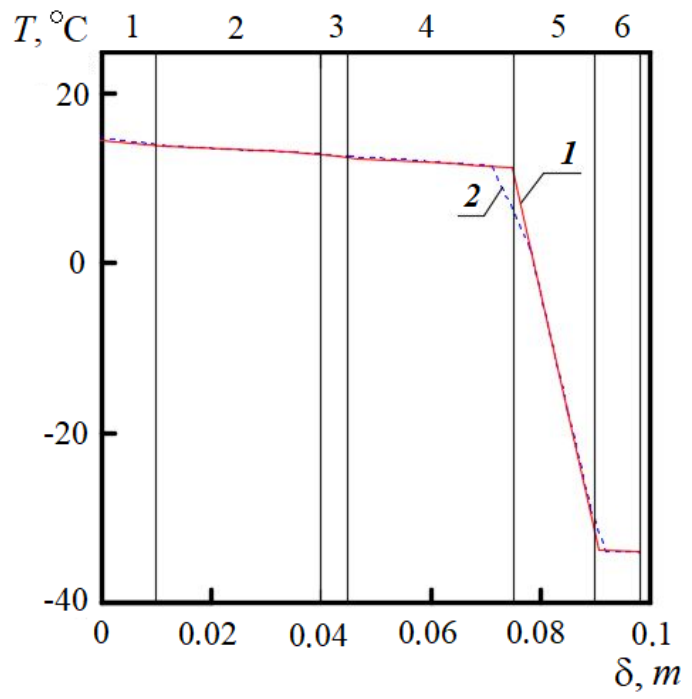

Fig. 3. Temperature distribution over the thickness of the fence (model number:

1 - approach 1;2 - approach 2

The temperature distribution (approach 2) is plotted over the section $y=1 \mathrm{~m}$. The graph shows that in layers 1-4 and 6 the temperature changes slightly, and in layer 5 (air) there is a sharp drop in temperature, which can be explained by the low thermal conductivity of air. A comparative analysis of temperature distribution graphs showed that the results of calculations using approaches 1 and 2 coincide, with the exception of narrow zones at the air-solid interface. This indicates that in this formulation the temperature field is onedimensional.

The Figure 4 shows the temperature distribution over the thickness of the panel of model No. 2. The number of grid elements is 54,500 . Analysis of the data obtained showed that the temperature decreases uniformly throughout the thickness of the enclosing structure. This indicates that the design is heat resistant, i.e. the body tends to maintain the original temperature. The thermal resistance of model No. 2 is $R=10.71 \mathrm{~m}^{2} \cdot \mathrm{K} / \mathrm{W}$. The temperature drop inside a separate layer occurs according to a linear law and does not depend on $y$.

Areas of low temperature change correspond to screens, and areas of temperature drop correspond to air gaps. The temperature drop in the air gap is significant, due to the low thermal conductivity of air and its width. 


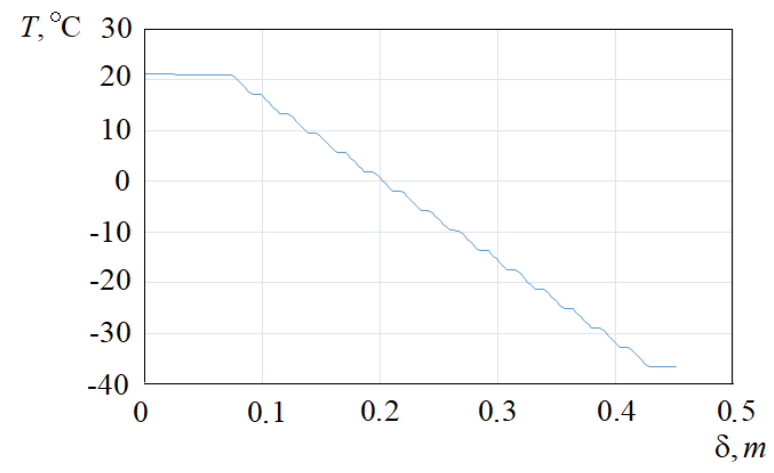

Fig. 4. Temperature distribution over layers of the building envelope of model No. 2

Next, we simulated the temperature field of model No. 3 in ANSYS Fluent. The number of grid elements is $1,497,600$. The size of the grid element in the air gap is $0.5 \mathrm{~mm}$.

As a result of the calculations, it was found that the natural convective movement of air is organized in air spaces. The air flow rises up along the warmer wall, and down along the colder one.

The temperature distribution in the upper section of the wall 1 and in the lower section 2 is shown in fig. 5, where it can be seen that the temperature distribution depends on the position of the section.

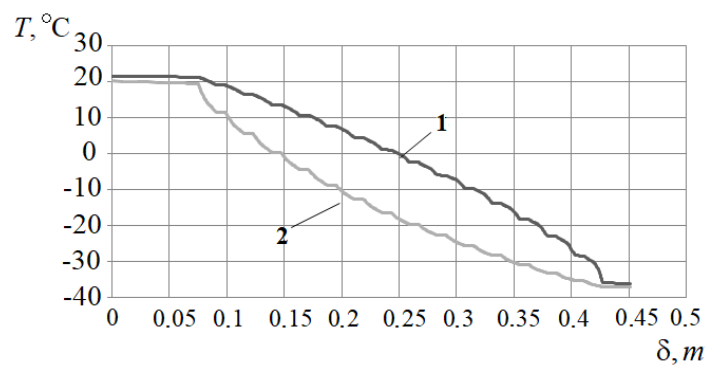

Fig. 5. Temperature distribution in sections 1 and 2 located in the upper and lower parts of the wall structure

Analysis of the temperature distribution showed that in the upper part of the structure the temperature is higher than in the lower one, which can be explained by the movement of air in the air spaces.

The next task was to select the optimal thickness of the air spaces. To solve the problem, the geometry of model No. 3 was used, in which the thickness of the air gap is a parameter. Were calculated models of enclosing structures with thicknesses of the air gap 8, 12, 16, 20, 24, $32 \mathrm{~mm}$. In addition, the dimensions (height and width) of the panel were changed: $0.5 \times 0.5 \mathrm{~m}, 1 \times 1 \mathrm{~m}, 2 \times 2 \mathrm{~m}$, and $3 \times 3 \mathrm{~m}$. The Figure 6 presents the calculated values of thermal resistance for different geometrical parameters. 


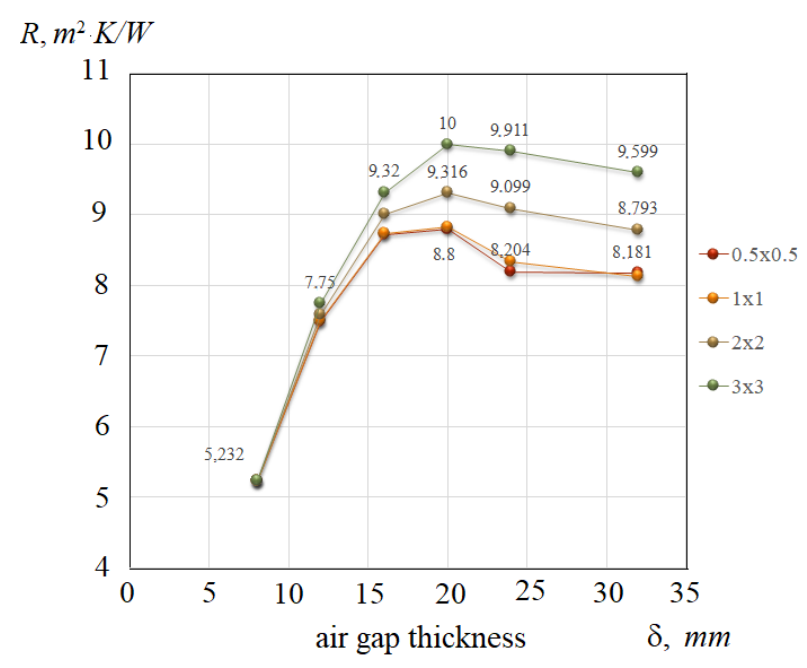

Fig. 6. Thermal resistances of the enclosing structure (model No. 2) depending on the thickness of the air gap and the size of the structure

The graph shows that with an increase in the thickness of the air gap from 8 to $16 \mathrm{~mm}$, the thermal resistance increases sharply and then stabilizes. This can be explained by a change in the structure of the air flow in the thickness of the air spaces. Due to the movement of air in the air gaps vortices are formed. As the thickness of the air layers increases, the partial vortices merge into one vortex, circulating at high speed between the heated and cooled surfaces, which leads to an increase in heat transfer. Thus, the optimum thickness of the air gap varies from 16 to $20 \mathrm{~mm}$. The thermal resistance of the enclosing structure is $R=9.32$ $\mathrm{m}^{2} \cdot \mathrm{K} / \mathrm{W}$, with an interlayer thickness of $16 \mathrm{~mm}$ and a construction size of $3 \times 3 \mathrm{~m}$. For a full study of heat transfer, a calculation was made taking into account radiant heat transfer. The fig. 7 shows the variation of the radiant heat flux $q_{l}$ in thickness of the enclosing structure with air gaps of $16 \mathrm{~mm}$.

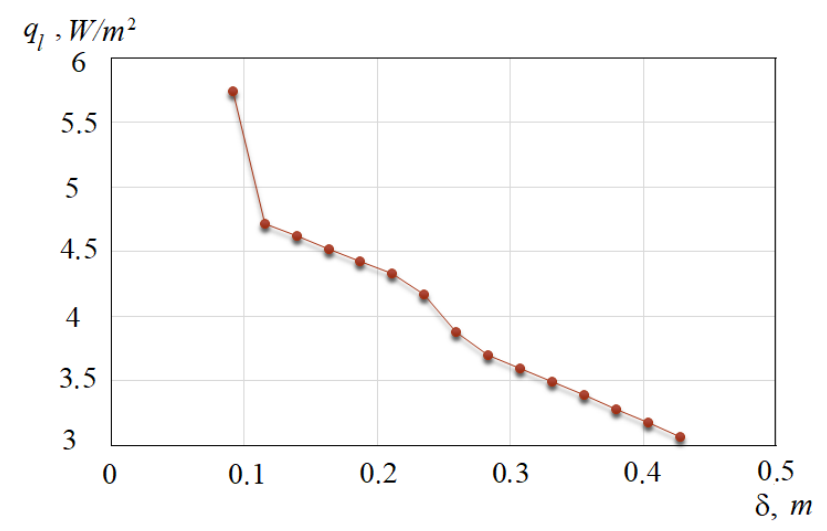

Fig. 7. Radiant heat flux through the thickness of the fence

Analysis of the data obtained shows that the intensity of the radiant heat flux decreases along the thickness of the structure, which is caused by the temperature variation over the layers of the structure. The total thermal resistance of the external enclosure (taking into account radiant heat exchange) calculated in ANSYS is $R=5.75 \mathrm{~m}^{2} \cdot \mathrm{K} / \mathrm{W}$. It should be noted that according to regulatory documents, the value of thermal resistance can be calculated 
only for one air gap, since there is no information on the temperature distribution inside the structure. When simulating with ANSYS, the data were obtained more accurately, since the temperature is directly calculated in each layer.

\section{Conclusions}

1. Traditional heat insulation materials in refractory structures can be replaced with shielded exterior refractories that use air gaps and shields.

2. The lack of thermal insulation material in shielded enclosing structures determines their durability and fire safety.

3. The optimum thickness of the air gap, at which the maximum thermal resistance is achieved, is $16 \div 20 \mathrm{~mm}$.

4. In a design with a small width of the air gap, the air is fixed or moves at a minimum speed, and therefore is a good heat insulator. With increasing width of the air gap - heat transfer increases due to the convective component.

5. Reducing the degree of blackness of the surface of the screens to $R=9 \mathrm{~m}^{2} \cdot \mathrm{K} / \mathrm{W}$ will allow to reach maximum values thermal resistance, i.e. at $\varepsilon=0.05$.

\section{References}

1. Fokin KF 2006 Construction heat engineering of the enclosing parts of buildings (Moscow: AVOK-PRESS) p 256

2. Chaplin VM 1905 The course of heating and ventilation (Moscow: GOSIZDAT) p 376

3. Danilov MN and Fedorova NN 2014 Numerical study of the heat-shielding characteristics of building sandwich panels Mag. Engineering Physics Journal T 87 No 5 pp 1096-1105

4. Kalitkin NN 1978 Numerical methods (Moscow: Science) p 512

5. Samarskiy AA and Gulin AV 1989 Numerical methods (Moscow: Science) p 432

6. Fletcher K 1991 Computational methods in fluid dynamics (Moscow: Mir) p 552

7. Siegel R 1992 Heat Transfer Hemisphere Publishing Corporation (Washington DC) p 178 